\title{
La diaspora : un levier pour le développement du tourisme en Haïti
}

The Haitian Diaspora: The way forward for the development of the tourism industry in Haiti

\section{Hugues Séraphin et Bénédique Paul}

\section{OpenEdition}

\section{Journals}

Édition électronique

URL : http://journals.openedition.org/tourisme/990

DOI : 10.4000/tourisme.990

ISSN : 2492-7503

\section{Éditeur}

Éditions touristiques européennes

\section{Référence électronique}

Hugues Séraphin et Bénédique Paul « La diaspora : un levier pour le développement du tourisme en Haïti », Mondes du Tourisme [En ligne], 11 | 2015, mis en ligne le 01 décembre 2015, consulté le 10 décembre 2020. URL : http://journals.openedition.org/tourisme/990 ; DOI : https://doi.org/10.4000/ tourisme.990

Ce document a été généré automatiquement le 10 décembre 2020.

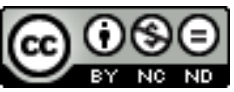

Mondes du tourisme est mis à disposition selon les termes de la licence Creative Commons Attribution - Pas d'Utilisation Commerciale - Pas de Modification 4.0 International. 


\section{La diaspora : un levier pour le développement du tourisme en Haïti}

The Haitian Diaspora: The way forward for the development of the tourism

industry in Haiti

Hugues Séraphin et Bénédique Paul

1 Il n'est pas aisé d'associer le phénomène de diaspora et le tourisme - le premier est marqué par la contrainte du départ et l'affliction, alors que le deuxième incarne l'intention et l'agrément (Saghi, 2011). Il est difficile aussi d'associer l'industrie touristique à la destination "Haïti" - d'aucuns ont oublié que cette destination a été, entre les années 1940 et 1960, une destination privilégiée dans la Caraïbe en matière de réception de touristes (Séraphin, 2014a). Haïti est davantage connue comme étant "lîle de toutes les tragédies" du fait du niveau de pauvreté de la population, des problèmes politiques et économiques constants, et enfin à cause des catastrophes naturelles qui l'ont touchée (Bonnet, 2010 ; Dupont, 2010). L'originalité de cet article réside justement dans cette association d'opposés que l'on pourrait même caractériser d'approche janusienne en référence au dieu Janus à deux visages (Dervin, 2010).

2 La diaspora représentant un marché lucratif pour une destination touristique (Hung, Xiao \& Yang, 2013), le ministère du Tourisme et des Industries créatives d'Haïti (MTIC) a fait de la diaspora haïtienne, depuis l'année 2011, une de ses cibles prioritaires pour la relance de l'industrie touristique du pays ${ }^{1} \mathrm{du}$ fait, entre autres, de la difficulté du gouvernement à attirer les investissements directs étrangers (Barreau, 2012) nécessaires au développement touristique et économique d'Haïti. Au vu de l'importance que revêt la diaspora pour le développement d'Haïti, il est primordial que les autorités locales aient une bonne compréhension de ses caractéristiques ainsi que de son potentiel économicotouristique réel (à la fois comme investisseur et/ou touriste) afin d'être en mesure de développer une stratégie adéquate pour la capter de manière efficace. 
3 En effet, ordinairement présentée comme une perte nationale en ressources humaines, la diaspora constitue pourtant un atout pour certains pays comme l'Inde, la chine ou les Philippines. L'exemple des diasporas résidant aux Pays-Bas a été documenté par Groot et Gibbons (2007) comme un creuset de ressources mobilisables. Ces auteurs ont montré qu'au lieu d'être un phénomène "brain drain" la diaspora peut représenter un "brain gain". Leurs résultats empiriques confortent la reconnaissance croissante des impacts positifs de la migration tant pour les pays d'accueil que pour les pays d'origine. Ils argumentent le fait que les diasporas peuvent être considérées comme des agents de développement (ibid. ). Dans le cas d'Haïti, la contribution de la diaspora représente une part importante du produit intérieur brut, des investissements directs étrangers ou de l'aide externe (Paul, 2008). Plusieurs auteurs s'accordent à dire que cette contribution va au-delà du financier pour toucher le développement de nouveaux marchés, le transfert de technologie, la philanthropie, le tourisme, la politique, le savoir, les nouvelles attitudes, mais aussi le changement culturel (Paul et Michel, 2013a, p. 61 ; De Haan, 2000 ; Meyer et Brown, 1999). Dans notre propos, nous nous focaliserons bien entendu exclusivement sur la question du tourisme.

Ce sujet de recherche relève d'une heuristique particulière dans la mesure où il existe un nombre limité de recherches académiques sur la question du tourisme en Haïti (Séraphin, 2014a). Les travaux scientifiques sur le rôle de la diaspora dans le développement touristique sont encore plus rares. Stéphanie Melyon-Reinette $(2009,2010)$ s'est beaucoup intéressée à la diaspora haïtienne d'un point de vue social (et ethnologique) sans pour autant établir un lien entre cette diaspora et son implication dans le développement économique du pays, et encore moins à son implication dans le développement de l'activité touristique. La même chose peut être dite de Bénédique Paul $(2008,2012 b)$. De même, Dautruche (2013) ; Doré (2010) ; Dupont (2009) ; Jules et Laplanche (2006) ; Théodat (2004) se sont intéressés au tourisme en Haïti sans s'être arrêtés sur la question de la diaspora et de son rôle potentiel dans ce secteur. Ainsi, dans la présente étude où nous considérons le tourisme diasporique comme un brain gain, nous proposons d'établir un lien entre les termes "tourisme" et "diaspora" qui n'ont rien d'incompatible dans le contexte haïtien.

5 En somme, cet article contribuera non seulement à la méta-littérature existante sur la question du tourisme en Haïti, mais surtout élargira la thématique en répondant à un certain nombre de questions jusqu'ici non abordées par les chercheurs qui s'intéressent au thème du tourisme en Haïti: Quel est l'impact de la diaspora haïtienne sur le développement de l'activité touristique? Face à tous les apports extérieurs, le tourisme en Haiiti saura-t-il garder son originalité ?

6 Au niveau méthodologique, notre étude qualitative repose essentiellement sur des recherches secondaires, plus précisément sur des travaux effectués par d'autres (Hammond et Wellington, 2013), couvrant des champs aussi divers que variés comme le tourisme en Haïti ; la diaspora et ses caractéristiques ; l'identité d'un territoire ; le capital humain; les méthodologies de recherches et le marketing. Cette méthode a pour avantage de fournir au(x) chercheur(s) une idée précise du contexte afin d'appréhender le problème dans sa globalité et de formuler des hypothèses (Silver, Stevens, Wrenn et Loudon, 2013). L'approche préconisée dans cette étude est quant à elle inductive et déductive au fur et à mesure que nous approchons de la conclusion.

7 Notre développement est divisé en trois parties : tout d'abord, dans "Haïti et sa diaspora", nous faisons une revue de littérature sur le sujet de la diaspora et de la diaspora haïtienne 
en particulier. Ensuite, "Contribution de la diaspora dans les investissements touristiques en Haïti" étudie le potentiel de la diaspora (comme touriste ou investisseur) comme source de développement touristique pour Haïti. La troisième partie intitulée "Haïti fait de la résistance positive" met en question la capacité de la destination à garder son authenticité. Dans la conclusion, nous mettrons en avant les limites de cette étude tout comme les opportunités et les raisons pour poursuivre d'autres études dans ce domaine.

\section{Haïti et sa diaspora}

\section{Les vagues migratoires de la diaspora haïtienne}

Les raisons qui poussent une personne à migrer sont complexes et multiples (BordesBenayoun, 2002; Dautruche, 2013; Melyon-Reinette, 2010 ; Cruse, 2012; Pocock \& McIntosh, 2013). Elles relèvent de données palpables et mesurables (différence de niveau de vie entre l'espace d'émigration et l'espace d'immigration, possibilités réelles d'emploi dans le pays d'immigration, danger pour le migrant dans son pays d'origine) et celles plus difficiles à appréhender qui appartiennent à la sphère des représentations (comme l'image véhiculée par les médias et par les migrants retournant dans leur pays d'origine). Il est important de mentionner que cette diaspora haïtienne choisit comme pays d'immigration l'Amérique latine, les Petites Antilles, la Guyane, la France, la République dominicaine, l'Afrique, le Canada, les États-Unis, Cuba, le Mexique. Sachant que les États-Unis, le Canada, les Petites Antilles et la France demeurent les premiers foyers d'accueil des migrants (Cruse ${ }^{2}$, 2012). En Haïti, comme dans beaucoup d'autres pays d'émigration, la contribution de la diaspora représente une part importante du produit intérieur brut, des investissements directs étrangers ou de l'aide. Cette contribution va au-delà de l'aspect financier pour toucher le développement de nouveaux marchés, le transfert de technologies, la philanthropie, le tourisme, la politique, le savoir, les nouvelles attitudes, mais aussi le changement culturel (De Haan, 2000 ; Meyer et Brown, 1999).

9 Ainsi, trois grandes vagues migratoires sont à distinguer dans l'histoire de la diaspora haïtienne. La première va de 1915 à 1935. La seconde, de 1965 à 1985, et enfin la dernière, de 2005 à 2014³. Ces trois vagues de la migration haïtienne correspondent à différentes étapes du tourisme en Haïti. Cela implique une expérience distincte pour l'industrie touristique dans le pays d'origine :

10 Ainsi, la première vague de la diaspora (1915-1935) correspond à une période où le tourisme ne consistait pas une activité économique pour le pays. En effet, la première administration du tourisme (office national du tourisme) apparait en Haïti en 1939. Cette génération n'est donc pas familière des activités de loisirs liées au tourisme en Haïti.

11 La deuxième phase de migration (1965-1985) est à cheval sur la dictature de François Duvalier (1957-1971) et de Jean-Claude Duvalier (1971-1986). La dictature de Jean-François Duvalier met un terme à l'activité touristique en Haïti, alors que sous la dictature de JeanClaude Duvalier on assiste à une reconstitution du tissu touristique de l'espace haïtien. Ce changement de politique en faveur du tourisme est caractérisé notamment par la construction de la route allant de Port-au-Prince à Jacmel (1976), par la construction de l'hôtel La Jacmélienne, par l'ouverture d'un Club Méditerranée (1981) sur la côte des Arcadins... À l'époque, les recettes du tourisme engrangées par Haïti dépassent de loin celle de sa voisine, la République dominicaine, dont l'activité économique est centrée sur 
l'industrie sucrière (Séraphin, 2010). Les ressortissants haïtiens de cette vague migratoire ont une expérience mitigée du tourisme en Haïti. Du fait des troubles politiques, la destination sort de la carte des destinations touristiques mondiales.

La dernière vague migratoire qui commence en 2005 (2005-2014), est marquée par des troubles politiques qui maintiennent la destination hors de la carte mondiale des destinations touristiques. Avec l'accession au pouvoir du président Martelly en 2011, Haïti gagne en stabilité politique. À ce climat de paix relative s'ajoutent les actions mises en œuvre par la ministre du Tourisme (Stéphanie Balmir Villedrouin), qui contribuent à remettre Haïti sur la carte des destinations mondiales du tourisme. Cette vague migratoire elle aussi a une expérience mitigée du tourisme en Haïti.

13 À ces différentes vagues migratoires doivent s'ajouter les descendants de ces primoémigrés afin que le champ de la diaspora soit entièrement couvert. Même s'ils n'ont pas le même attachement à la terre natale que leurs parents, ils n'en sont pas pour autant indifférents. Il n'en demeure pas moins que le retour au pays de cette seconde génération va s'avérer difficile. Comme le souligne Elbaz (2010a), la création d'Israël n'a pas causé le retour de la majorité des juifs du monde entier vers cet État, pas plus que l'abolition de l'esclavage n'a causé le retour de tous les Noirs vers l'Afrique.

Ces cas de figures montrent bien qu'il y a un lien entre les différentes vagues migratoires et le tourisme (et plus précisément l'expérience touristique). La période du départ influant très certainement sur la perception du tourisme dans le pays d'origine. Les deux sous-parties suivantes nous permettront de répondre à la question suivante : Est-ce qu'un fort sentiment d'identité nationale peut contribuer au développement du tourisme diasporique?

\section{Diaspora haïtienne et identité}

Pour bien comprendre l'expression identitaire dans la diaspora, il y a lieu de distinguer les deux générations actrices de cette expression. À l'intérieur même du phénomène de diaspora on distingue, d'un côté, les primo-arrivants, qui cultivent une certaine nostalgie du pays et le revisitent en pèlerinage ; de l'autre côté, il y a la jeune génération pour qui le territoire d'origine s'est éloigné. Cette génération ne cultive ni nostalgie ni espoir de retour (Bordes-Benayoun, 2002). Elle vit alors, selon Elbaz (2010a), une sorte d'hybridation. En d'autres termes, il n'y a pas d'oubli de la culture d'origine, mais une forte intégration dans un mouvement identificatoire qui prend en compte la culture d'accueil. Selon Elbaz (2010b), parler de "dédiasporisation" serait une erreur, car dépendamment de la raison et des conditions de l'émigration, les liens avec le pays d'origine sont maintenus plus ou moins fortement. Dans le cas d'Haïti, où l'émigration politique fut progressivement remplacée par l'émigration économique (Paul, 2008), la conservation des liens avec le territoire d'origine se manifeste à travers la construction et le développement de communautés (Dufoix, 2003). Ces dernières sont créatrices sinon conservatrices de liens entre une population sur plus d'un territoire. Ainsi, dans une recherche sur l'identité haïtienne publiée en 2009 par Bénédique Paul, il a été souligné le fait que l'haïtianité est très fortement exprimée dans les communautés haïtiennes de la diaspora (Paul, 2009).

Pour l'anthropologue Maud Laëthier qui analyse la diaspora haïtienne de la Guyane française, l'expression identitaire apparaît sous trois configurations: une première configuration liée à des stigmatisations, une deuxième qui montre une unité 
communautaire et une troisième visant l'intégration (Laëthier, 2007). Bénédique Paul (2009) qualifie cette expression identitaire d'“haitianité". Cette dernière a pris un sens très fort au sein de la diaspora haïtienne tantôt comme nécessité d'être soi-même dans un contexte d'exclusion, tantôt comme besoin de nourrir les liens avec le pays. En effet, la diaspora est impliquée, pour des raisons de mémoire (Laëthier, 2006) et d'insertion (Audebert, 2006), dans le façonnement de deux territoires (d'origine et d'accueil). L'analyse menée ici relève, selon Denis-Constant Martin et le groupe IPI (Identité, pouvoirs et identifications), d'une approche peu documentée qui considère le rôle économique de l'identité, notamment en matière de formes du développement économique territorial (Martin, 2010).

L'expression identitaire dans la diaspora haïtienne, comme dans d'autres diasporas, s'est progressivement structurée autour d'une motivation économique et dépasse les stéréotypes traditionnels. Loin d'être une expression de simple patriotisme ou de nationalisme, elle est devenue une volonté et la traduction concrète de celle-ci en des termes économiques dont les retombées sont désormais visibles à travers la réussite professionnelle, notamment dans le cas de la diaspora haïtienne des États-Unis et du Canada ; à travers les nombreux petits projets sur le territoire haïtien qui ont été mis en œuvre à l'initiative des individus ou des organisations de la diaspora. Ces projets sont d'envergure soit collective (école, route, centre de santé, etc.), soit individuelle (construction de maisons, création de micro-entreprises dans le domaine du tourisme: hôtels, cafés, restaurants, services connexes et annexes). Ils semblent contribuer, dans une certaine mesure, à refaçonner le territoire d'origine qui devient de plus en plus un mélange entre l'autochtone et l'importation culturelle diasporique. La question qui se pose alors, et ce dans une logique de développement décentralisé d'Haïti est la suivante : dans quelle mesure l'haïtianité, très forte au sein de la diaspora, peut-elle être mobilisée comme vecteur de développement territorial en Haïti, et plus particulièrement dans le secteur du tourisme? Cette question est débattue dans la section suivante.

\section{Diaspora haïtienne et développement territorial}

18 Les Haïtiens ont reçu de leur diaspora, en 2012, quelque 1,988 milliard de dollars américains contre 2,057 milliards en 2011. En 2014, selon les données officielles de la Banque mondiale, le montant de ces transferts est aussi élevé. D’un point de vue microéconomique, et de manière générale, les transferts de fonds représentent une source importante de revenus pour des millions de familles qui les reçoivent, même dans les pays ayant un produit intérieur brut (PIB) élevé. En Haïti, ces transferts contribuent largement à lisser la consommation des bénéficiaires régulièrement touchés par des crises d'origine politique ou naturelle (Orozco, 2006; Paul, 2008). Ils représentent une part importante du PIB d'Haïti et dépassent parfois les investissements directs étrangers ou l'aide externe. Ce qui en fait un potentiel considérable de développement, notamment lors des périodes de crises.

La diaspora haitienne peut véritablement être considérée comme un lieu d'élaboration et de financement d'activités génératrices de fonds pour Haïti. Il a été argumenté que la contribution de la diaspora haïtienne, par ses caractéristiques anticycliques et non contractuelles (Paul, 2008), est une source financière particulièrement précieuse pour Haïti, qu'il convient de mobiliser de manière efficace et intelligente (Dandin, 2012 ; Paul, 2012b). Par exemple, la crise économique de 2008-2009 avait réduit les flux de transferts 
effectués par la diaspora vers Haïti, mais la solidarité provoquée par le séisme du 12 janvier 2010 a permis d'accroître ces transferts à un rythme encore plus élevé pour atteindre presque 2 milliards de dollars en 2014 selon les données officielles (voir figure 1 ci-dessous). Selon la Banque mondiale, ces transferts représentent $21,1 \%$ du PIB d'Haïti.

Figure 1. Évolution croissante des transferts de fonds issus de la diaspora haïtienne

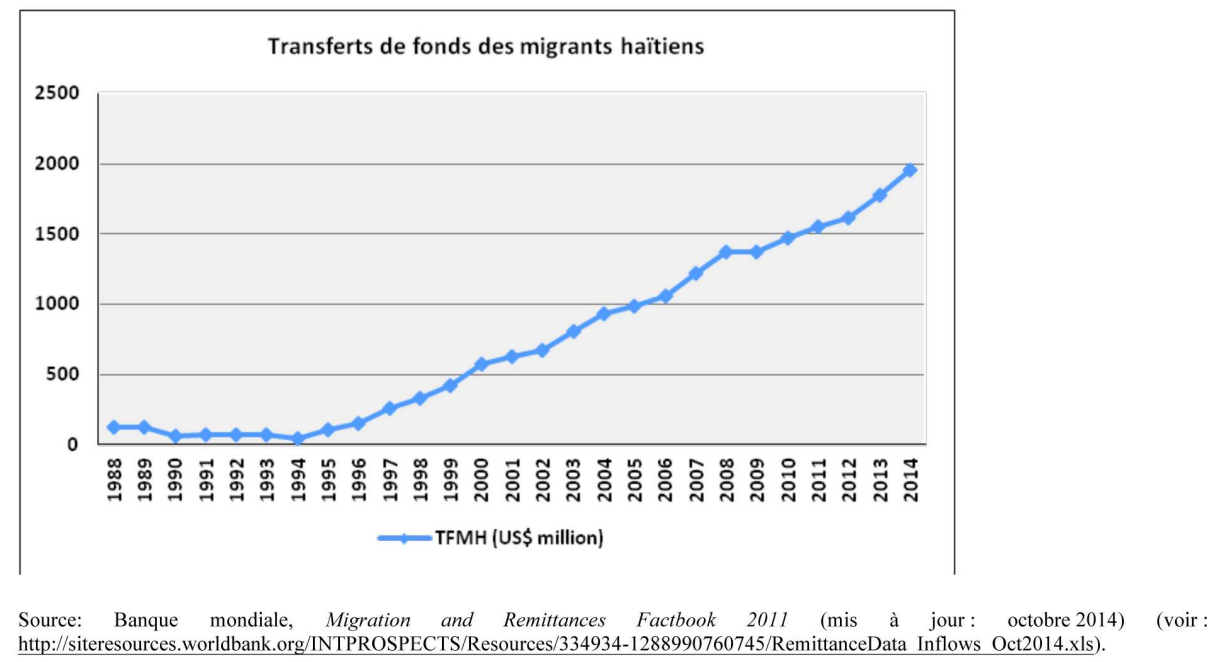

En effet, la diaspora haïtienne produit des externalités positives tant pour le pays d'accueil que le pays d'origine. Dans un article sur les externalités diasporiques et les transferts technologiques, Lodigiani (2008) montre, à partir de données de panel pour 92 pays, incluant des nations développées et en développement dont Haïti, pour les années 1980 à 2000, que la migration stimule la croissance dans les pays éloignés de la frontière. Ses résultats confirment ceux de Vandenbussche, Aghion et Meghir (2006) qui étudient la contribution du capital humain à la croissance économique.

La diaspora constitue incontestablement une solution aux problèmes de pauvreté en Haïti. Le développement économique d'Haïti passe par une mobilisation de ses ressources propres. Parmi ces dernières, les plus précieuses sont ses ressources humaines qualifiées. Même si une grande majorité de celles-ci se trouvent en dehors du territoire national, pour peu que les liens soient conservés et traductibles en des opportunités, il y va de la stratégie de chercher à impliquer au mieux la diaspora dans le développement territorial. L'argumentation de Paul et Michel (2013a, 2013b), continuant Barré et al. (2003), sur la mobilisation de la diaspora académique haïtienne est un exemple de stratégie capable de transformer le brain drain en brain gain dans l'enseignement supérieur en Haïti.

La plus grande enquête disponible menée par le Fonds multilatéral d'investissement (Fomin) de la Banque interaméricaine de développement (BID) a révélé que les transferts de fonds effectués par les migrants haïtiens (TFMH) sont effectués en de petits montants. Ainsi, ils peuvent être difficilement mobilisés dans le cadre de grands programmes nationaux. Cependant, à l'échelle territoriale, nous soutenons l'idée qu'une meilleure canalisation de cette contribution est possible. Tout d'abord, les migrants se réunissent souvent en associations et maintiennent des liens avec les territoires d'origine. Ces organisations offrent des opportunités de financement de projets structurants à l'échelle territoriale. 
L'enquête de la BID n'avait pas pris en compte l'investissement important et croissant de la diaspora haïtienne dans la construction et dans les entreprises en Haïti. Même si les données agrégées sur ces deux types de contributions ne sont pas disponibles, elles ont sans doute un impact transformateur visible sur le territoire national, notamment à partir de l'année 2010. Il suffit pour les administrateurs publics territoriaux de faire preuve d'une meilleure gestion publique territoriale capable de mettre en confiance la diaspora qui souhaite contribuer au développement de leur lieu de naissance. Par exemple, tandis que les transferts réguliers issus de la diaspora haïtienne visent à compenser l'absence de sécurité sociale (retraite ou subvention pour les personnes non actives économiquement), il est possible d'envisager la création de mutuelles de solidarité formelle avec la participation des migrants. Une autre piste est de faire de la diaspora un acteur actif du tourisme local soit en tant qu'investisseur, visiteur et/ou employé. C'est d'ailleurs sur cette piste que nous allons nous attarder dans la partie suivante. Cependant, impliquer cette diaspora ne semble d'ailleurs pas être une option pour Haïti à plus d'un titre. D'une part, si Haïti ne le fait pas, elle sera obligée de faire venir de la main-d'œuvre qualifiée étrangère, car cela lui prendra plus de soixante-dix ans pour produire une masse de diplômes équivalente à celle déjà disponible au niveau de la diaspora haïtienne vivant aux USA (Wah, 2013). D'autre part, les revenus générés par ce groupe sont plus importants pour le secteur du tourisme en Haïti (ibid.). En dernier lieu, il semblerait que les initiatives menées par la diaspora sont celles qui ont le plus été couronnées de succès du fait de la bonne connaissance du terrain de ces derniers et surtout de leur volonté profonde d'aider (ibid.).

\section{Contribution de la diaspora au secteur touristique en Haïti}

\section{Préambule}

24 L'instabilité politique qui a suivi l'ère des Duvalier (1957-1986) a porté une atteinte sérieuse au tourisme en Haïti qui, petit à petit, a disparu de la carte des destinations mondiales (Séraphin, 2014b). Cet état de fait dans le secteur du tourisme correspond également à une vague massive de départs d'Haïtiens vers d'autres cieux soit pour échapper à des représailles (motif politique), soit pour améliorer leurs conditions de vie (motif économique). Heureusement, les autorités haïtiennes actuelles en charge du tourisme ont très bien compris l'importance de cette diaspora puisque la ministre du tourisme a multiplié les actions de séduction envers la communauté haïtienne vivant aux États-Unis et au Canada. L'amélioration significative des conditions sociopolitiques et économiques d'Haïti peut contribuer au retour progressif d'une partie significative de la diaspora. En effet, Lewis (1998) et Pellegrino (2000) expliquent qu'à l'intérieur d'un même territoire les pauvres peuvent décider de quitter la campagne pour la ville dans l'objectif d'améliorer leur condition de vie. Mais le retour au point de départ est considéré dès que des opportunités économiques se présentent (Paniagua, 2002). Même si ce n'est pas forcément un mouvement identique qui se fait dans le cas d'une migration extra territoriale, on constate depuis peu un retour timide de la diaspora haïtienne pour effectuer notamment des investissements dans le secteur du tourisme et plus particulièrement dans l'hôtellerie-restauration ( $c f$. tableau 1). Pour Jerry Tardieu, P-DG de 
l'hôtel Oasis (premier hôtel de standard international en Haïti qui a ouvert ses portes le 12 décembre 2012), "investir et s'investir en Haïti est un acte de foi"."

Tableau 1. Hôtellerie et diaspora en Haïti

\begin{tabular}{|l|l|}
\hline \multicolumn{1}{|c|}{ Hôtels } & \multicolumn{1}{c|}{ Dates d'ouverture } \\
\hline Hôtel Oasis & 2012 \\
\hline La Lorraine & 2012 \\
\hline Hôtel Best Western & 2013 \\
\hline Villa Nicole & 2013 \\
\hline Hôtel La Source & 2013 \\
\hline Monte Cristo & 2014 \\
\hline Colin Hôtel & 2014 \\
\hline
\end{tabular}

Source : Séraphin (2014a)

\section{Pourquoi la diaspora comme cœur de cible}

Tous les éléments que nous avons recueillis semblent indiquer que la diaspora est le cœur de cible idéal pour le développement durable du tourisme en Haïti. Une analyse des forces, faiblesses, opportunités et menaces (SWOT) de ce groupe nous permettra d'analyser sa plus-value par rapport à d'autres groupes ( $c f$. tableau 2). Pour ce faire, nous nous appuierons sur les travaux de Séraphin (2014b), Wah (2013), Jadotte (2012), Newland et Taylor (2010).

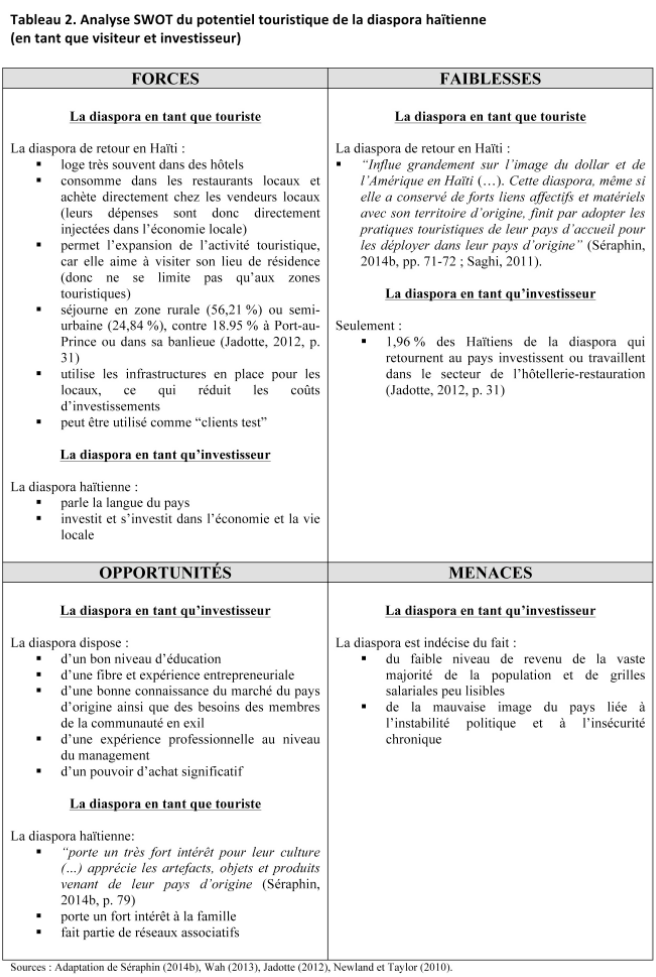

Notre étude montre que la diaspora représente une véritable aubaine pour le tourisme en Haïti. Les forces et les opportunités sont de loin supérieures aux menaces et faiblesses. Toujours selon l'étude, les membres de la diaspora haïtienne représentent avant tout un atout en tant que touristes (et non en tant qu'investisseurs ou travailleurs du secteur du 
tourisme). Une étude comparative reposant sur les mêmes sources montre que ce groupe est beaucoup plus intéressant que les touristes non issus de la diaspora (cf. tableau 3).

Tableau 3. Approche comparative tourisme diasporique et non diasporique en Haïti

\begin{tabular}{|l|l|}
\hline \multicolumn{1}{|c|}{ Tourisme diasporique docal (hôtels, } & \multicolumn{1}{|c|}{ Tourisme non diasporique } \\
\hline $\begin{array}{l}\text { Consomme davantage dans des chaînes d'hôtels qui sont } \\
\text { restaurants, souvenir } \\
\text { souvent intégrées dans des enclaves }\end{array}$ \\
\hline $\begin{array}{l}\text { Dépenses injectées directement dans } \\
\text { l'économie locale }\end{array}$ & $\begin{array}{l}\text { Dépenses repartent dans le pays d'origine. } \\
\text { Peu de connexion avec l'économie locale }\end{array}$ \\
\hline $\begin{array}{l}\text { Permet la répartition de l'activité touristique } \\
\text { sur l'ensemble du pays, car la diaspora aime } \\
\text { visiter son lieu de résidence }\end{array}$ & $\begin{array}{l}\text { Se cantonne à la visite des grands centres } \\
\text { touristiques }\end{array}$ \\
\hline $\begin{array}{l}\text { Utilise les infrastructures en place pour les } \\
\text { locaux, ce qui réduit les coûts } \\
\text { d'investissements et les frais d'entretien }\end{array}$ & $\begin{array}{l}\text { Est consommateur de ressources qui } \\
\text { entrainent des investissements lourds }\end{array}$ \\
\hline $\begin{array}{l}\text { En cas de troubles dans le pays d'origine, la } \\
\text { diaspora est plus à même d'à analyser } \\
\text { l'ampleur de la situation }\end{array}$ & $\begin{array}{l}\text { Très influencé par les médias pour leur choix } \\
\text { de vacances }\end{array}$ \\
\hline
\end{tabular}

Sources : Adaptation de Séraphin (2014b), Wah (2013), Jadotte (2012), Newland et Taylor (2010)

Si la diaspora représente un atout majeur pour le développement du tourisme en Haïti, on sait que cette diaspora, même si elle a conservé de forts liens affectifs et matériels avec son territoire d'origine, finit par adopter les pratiques touristiques de son pays d'accueil pour les déployer dans son pays d'origine (cf. tableau 4).

Tableau 4 : La diaspora haïtienne dans le monde

\begin{tabular}{|c|c|c|c|c|c|c|}
\hline \multicolumn{7}{|c|}{$\begin{array}{l}\text { NUMBER OF HAITIAN MIGRANTS BY DESTINATION COUNTRY, } 2010 \\
\text { Number of individuals and percentages }\end{array}$} \\
\hline & $\begin{array}{l}\text { Haitian destination } \\
\text { countries }\end{array}$ & $\begin{array}{c}\text { Number of } \\
\text { Haitian } \\
\text { migrants }\end{array}$ & $\begin{array}{c}\text { Structure } \\
(\%)\end{array}$ & $\begin{array}{c}\text { Destination } \\
\text { countries }\end{array}$ & $\begin{array}{c}\text { Number of } \\
\text { Haitian } \\
\text { migrants }\end{array}$ & $\begin{array}{c}\text { Structure } \\
(\%)\end{array}$ \\
\hline 1. & United States & 587,149 & 54.15 & 12. Suriname & 869 & 0.08 \\
\hline 2. & Dominican Republic & 311,969 & 28.77 & 13. Spain & 769 & 0.07 \\
\hline 3. & Canada & 73,753 & 6.80 & 14. Germany & 645 & 0.06 \\
\hline 4. & France & 42,103 & 3.88 & 15. Mexico & 500 & 0.05 \\
\hline 5. & Bahamas & 19,051 & 1.76 & 16. Italy & 278 & 0.03 \\
\hline 6. & Netherlands Antilles & 3,757 & 0.35 & 17. United Kingdom & 217 & 0.02 \\
\hline 7. & Belgium & 1.921 & 0.18 & 18. Denmark & 177 & 0.02 \\
\hline 8. & Venezuela & 1.509 & 0.14 & 19. Sweden & 138 & 0.01 \\
\hline 9. & Switzerland & 1,257 & 0.12 & 20. Greece & 131 & 0.01 \\
\hline 10. & The Netherlands & 1.087 & 0.10 & Other countries & 36,027 & 3.32 \\
\hline 11. & Cuba & 909 & 0.08 & TOTAL & $1,084,216$ & 100.00 \\
\hline
\end{tabular}

Les États-Unis étant la première destination d'immigration des Haïtiens, nous nous interrogerons dans la dernière partie sur l'impact potentiel que ce fait peut avoir sur le tourisme en Haïti, surtout si on ajoute à cela que les touristes américains confirment en 2010 leur statut de premier pays fournisseur de touristes en Haïti (Séraphin, 2014b). Près de trois quarts des arrivées de la catégorie des touristes de séjour, soit 71,94\%, proviennent des États-Unis d'Amérique. Cela s'explique non seulement par la proximité d'Haïti avec ce pays, mais aussi par l'importance de la communauté haïtienne résidant aux États-Unis (MTIC, 2011). En partant du principe que toutes les destinations se battent pour les mêmes touristes, et que la tendance est à aller aux devants des besoins du touriste depuis les années 1970 (Séraphin, 2011), notre hypothèse est donc la suivante : 
On assistera sur le long terme à une américanisation des prestations et services touristiques en Haïti.

\section{Haïti fait de la résistance positive}

\section{Globalisation ou "glocalisation" ?}

Les processus d'acculturation et du langage sont étroitement liés. La langue anglaise s'est au cours des dernières années imposées notamment dans le secteur du tourisme au niveau des transactions. Elle s'impose de plus en plus comme la langue principale du tourisme (Korstanje, 2012). En suivant la démarche de Korstanje (ibid.), on pourrait penser que le tourisme haïtien s'anglicise. Dans son étude de cas intitulée The Influence of Languages in the Travel Agencies Name: The Case of Buenos Aires ${ }^{5}$, Korstanje (ibid.) cherche à déterminer le niveau d'acculturation du secteur du tourisme à destination de Buenos Aires en s'appuyant sur l'étude des enseignes des agences de voyages et hôtels. Après avoir analysé 1050 enseignes, il constate qu'une majorité d'hôtels ont un nom à consonance locale du fait que ces derniers sont à majorité possédés par des locaux. Le cas du tourisme haïtien n'est pas différent.

En lisant Thomson (2004 [1991]), on a la forte impression que les Haïtiens ont un certain penchant pour la langue anglaise. Bram (1967) souligne quant à lui l'importance pour les sociétés d'adopter les langues internationales afin de se connecter au reste du monde. À quel point ces affirmations se vérifient-elles dans le secteur du tourisme (hôtellerie) en Haïti? En nous appuyant sur la liste des hôtels d'Haïti (6 hôtels ont été pris en considération) qui figure sur le magazine [en ligne] Magic Haïti, il en ressort que seulement $5 \%$ des hôtels ont une dénomination anglo-saxonne et que 0,6 \% des hôtels ont une dénomination hispanique. Ces données reflètent la répartition de la diaspora haïtienne dans le monde ( $c f$. tableau 4), avec les États-Unis (langue anglaise) en tête des destinations, suivis de la République dominicaine (langue espagnole). Ces données reflètent également la faiblesse de l'actionnariat étranger dans le secteur de l'hôtellerie en Haïti. En effet, les propriétaires des hôtels sont essentiellement : a) soit des Haïtiens de naissance (exemple du Club Indigo, ex-Club Med, ou encore du Karibe Hotel) ; b) soit des étrangers installés en Haïti depuis de nombreuses années (cas du Club Indigo, ou encore de l'hôtel Florita). Ces derniers se considèrent, du fait de leur ancienneté dans le pays, comme étant des Haïtiens. C'est dire que dans le tourisme en Haïti, et plus particulièrement dans le secteur de l'hôtellerie, il y a un fort ancrage local. Autrement dit, Haïti réussit plus ou moins à conserver son identité malgré le développement du tourisme.

31 Il est évidemment possible de se demander si cet ancrage pourra perdurer dans le temps, du fait des projets de développement du tourisme du gouvernement actuel. La volonté gouvernementale se traduit à travers le slogan "Haiti is open for business". Avec cette ouverture, Haïti saura-t-elle garder son identité et toujours mettre en avant les figures et mises en scène d'événements fondateurs afin de maintenir le sentiment d'appartenance commune (Pulvar, 2006) ?

Dans l'optique de la conservation de l'identité haïtienne dans le secteur du tourisme et plus particulièrement celui de l'hôtellerie, la tension se situe pour le gouvernement dans le choix à faire entre une hôtellerie haïtiano-haïtienne et une hôtellerie de chaînes (internationales). En même temps, l'intérêt national pour le développement de 
l'entrepreneuriat local est un phénomène nouveau qui ne peut être négligé. Privilégier l'entrepreneuriat haïtien dans le secteur du tourisme serait avant tout faire preuve de pragmatisme. Car, selon Tracy Kidder, les seuls success stories d'actions mises en place en Haïti par les ONG et la communauté internationale ont été des programmes ou les Haïtiens ont été aux commandes (Kidder, 2010). C'est pourquoi des voix se sont élevées pour dire qu'Haïti ne doit plus être un "peuple objet (...), le peuple haitien doit reprendre la reconstruction du pays en main (...), seules les mains magiciennes du peuple pourront sortir le pays du désastre moral, social et économique" (Kidker, ibid.).

Le développement, en matière touristique, doit rechercher l'adhésion de la population. L'étude de Thernil (2004), intitulée Perceptions of Haitians toward Tourism Development in Rural Haiti, montre que les Haïtiens ont un avis plutôt mitigé sur la question du développement du tourisme dans leur pays. Afin de rallier la population à la cause du tourisme, la mobilisation des acteurs locaux paraît nécessaire. De la même façon, dans cette dynamique, la diaspora haïtienne, par son attachement territorial, constitue un atout dans la transformation pro-touristique des territoires sans nécessairement produire de l'acculturation, tout en ramenant les chaînes internationales en Haïti. Car, même s'il est indéniable que les Haïtiens ont la capacité de faire avancer les choses, il n'en demeure pas moins que la présence de l'hôtellerie de chaîne internationale est importante pour l'image de la destination et la réussite de la démarche de marketing touristique international entamée depuis deux ans par le gouvernement haïtien.

Autrement dit, la préférence nationale ou locale en matière d'investissement touristique n'est pas totalement justifiée. La présence des chaînes hôtelières internationales a mis fin à la tendance d'avant la fin des années 1960 qui voyait l'hôtellerie comme un commerce détenu et opéré principalement par des entrepreneurs privés, c'est-à-dire des individus et quelques familles (Larivière et Jussaume, 2004, p.11). Mais l'alternative, c'est-à-dire l'exploitation par des entrepreneurs haïtiens d'enseignes internationales, n'a pas toujours réussi. Par exemple, le cas de l'Holiday Inn en Haïti (redevenu le Plaza) n'a pas été une tentative concluante. Cependant, l'intérêt actuel pour des investissements mixtes (Haïtiens et étrangers) offre une porte d'entrée intéressante pour canaliser la contribution de la diaspora haïtienne vers le secteur touristique. Cela est d'autant plus vrai que la diaspora elle-même constitue le noyau dur des flux touristiques vers Haïti, comme dans d'autres pays de la région tels la République dominicaine, Le Salvador, Honduras, Nicaragua et le Mexique (Orozco, 2004). Toutefois, dans un objectif de conservation de l'identité des territoires (Faure, 2010), ce qui est en grande partie la raison de leur attractivité, la participation de la diaspora doit faire partie d'un développement territorial contrôlé.

\section{Vers un développement hybride du tourisme en Haïti}

La diaspora haïtienne, qui fut dans les années 1970-1980 mise à l'écart du processus de développement national, a progressivement repris les liens de sang et de symbole qui la lie avec le territoire d'origine. Elle se pose désormais en acteur incontournable dans le développement touristique d'Haïti. Cependant, dans le cadre d'une volonté de développer le tourisme, il est important que le gouvernement haïtien fasse en sorte que le degré d'influence extérieur sur le tourisme demeure modéré afin que le secteur garde son originalité. Notre argumentation du rôle potentiel de la diaspora haïtienne dans le développement du tourisme en Haïti dans une optique de développement territorial est 
fondée sur l'idée de la conservation de l'identité des territoires. En effet, si l'on part du principe que les Américains ont la réputation d'être des conquéreurs connus pour leur ingérence dans la politique d'autres pays ; que la seconde génération d'Haïtiens aux ÉtatsUnis représente un groupe de 340000 personnes $^{6}$; et enfin que ce groupe a développé une nouvelle forme d'haïtienneté dite "rhizomique hybride" (Melyon-Reinette, 2010), cette nouvelle génération peut malgré elle encourager une métamorphose de l'activité touristique en Haïti (cf. figure 2). L'hôtel Oasis en est l'exemple même. L'hôtel, exploité sous la forme de franchise est dirigé et détenu par un actionnariat issu de la diaspora (installé au préalable aux États-Unis). Le Best Western suit également un modèle similaire. Est-ce la fin d'un secteur touristique contrôlé exclusivement par des Haïtiens (non issus de la diaspora)? En effet, comme toutes les petits États insulaires, Haïti demeure très vulnérable (Angeron et Saffache, 2008). Fort de ces constats, il s'agit donc de mesurer l'attachement de la diaspora à son territoire d'origine (Bruneau, 2006), notamment en matière d'investissements touristiques (de Villanova, 2007).

Figure 2. Changement de nature du secteur touristique (hôtelier) haïtien
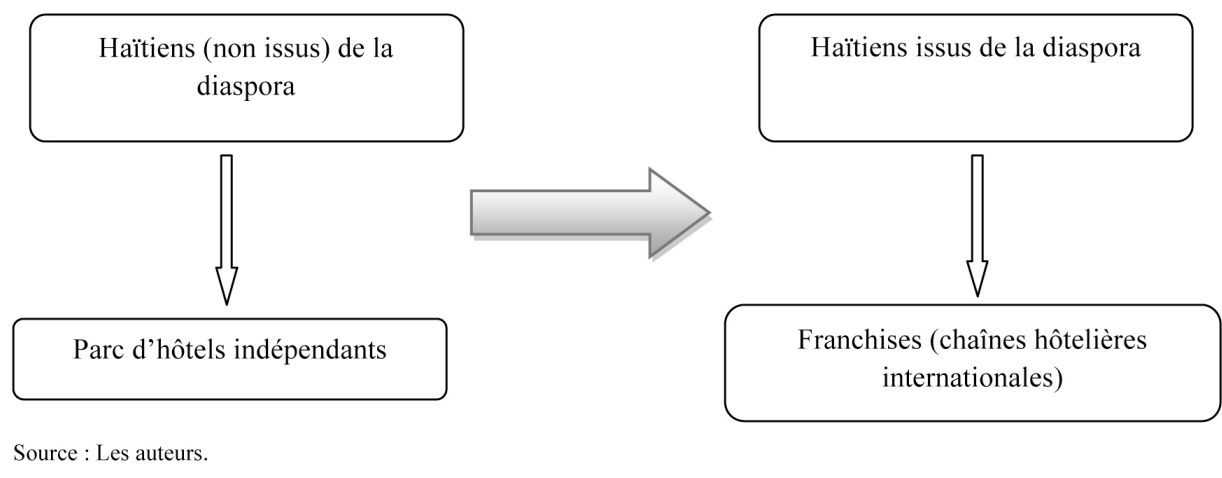

\section{Conclusion}

Comme dans le cas des Burkinabè (Bertoncello, 2010), les Haïtiens de la diaspora (après avoir été tenus à l'écart) sont désormais considérés comme les acteurs incontournables pour le développement du pays. Le développement d'une conscience d'appartenance commune (Pulvar, 2006) afin que les Haïtiens de la diaspora aient l'impression d'être des “citoyens à part entière" et non des citoyens entièrement à part (Dewitte, 2002) s'est révélé être très important. Le retour de cette diaspora qui est encore timide ne se fait pas non plus sans peine. En effet, les hommes n'évoluent pas dans l'espace tel qu'il est, mais plutôt dans l'espace tel qu'ils le perçoivent (Dubesset, 2012). Toujours dans ce même ordre d'idées, les recherches de Pocock et McIntosh (2013), montrent que les personnes qui ont vécu loin de chez elles ont une perception et une expérience différentes du retour. Certains en ont une représentation idyllique et parfois déchantent de ce retour, d'autres en dépit des difficultés parviennent à s'intégrer. Le challenge du ministère du Tourisme d'Haïti est de redonner ou de donner envie à la diaspora de venir passer ses vacances en Haïti et/ou d'investir dans le secteur du tourisme. La propension des membres de la diaspora souhaitant venir en vacances au pays (étant plus importante que celle qui souhaite investir ou travailler dans le tourisme), il semble important d'adopter une stratégie marketing adaptée, car la diaspora ne constitue pas un groupe homogène. De ce 
fait nous suggérons une segmentation sur la base des générations (primo-arrivants ou enfants des primo-arrivants) et du souvenir du pays d'origine (vagues migratoires). La finalité de la manœuvre est de regrouper ces derniers en cluster en fonction de leurs besoins avec pour objectif de différencier l'offre de produits et/ou de services (Blythe, 2009).

La prise en compte du rôle de la diaspora est fondée sur la nécessité d'associer toutes les constituantes de la société haïtienne dans la démarche de développement touristique. Dans cette logique, la population locale constitue également un acteur insuffisamment impliqué dans le développement touristique. Pourtant, elle est récipiendaire d'une partie considérable des transferts de fonds issus de la diaspora. La population locale ne doit pas être appréhendée uniquement comme main-d'œuvre dans les établissements relevant du tourisme. Elle peut être également consommatrice. Car le tourisme est souvent l'une des premières choses auxquelles les nouveaux consommateurs aspirent (Duthion et Michaud, 2011). Il est empiriquement démontré, dans une étude de cas menée à Samoa, que le tourisme intérieur peut avoir des avantages économiques, socio-culturels et politiques importants et qu'il ne devrait pas être traité en "poor cousin" (Scheyvens, 2007).

Aujourd'hui il est clairement reconnu que la compétitivité est un indicateur de performance. Dans une prochaine étude, il serait intéressant de proposer un cadre analytique pour évaluer et comparer le potentiel touristique de la diaspora haïtienne et de ses principaux concurrents de la Caraïbe. À chaque destination serait attribué un score qui tient compte des caractéristiques associées au potentiel touristique de la diaspora dans chaque territoire. Potentiel qui, pour son évaluation, prendrait appui sur les tendances évolutives récentes en termes d'implication de la diaspora en tant que touriste, en tant que visiteur et en tant que travailleur; et enfin l'impact de la promotion touristique. Ce faisant, des indices de potentiel touristique relatif seraient élaborés pour chacune des 28 destinations de la Caraïbe composant l'échantillon, indices mesurés par la moyenne des scores réalisés ainsi que par une analyse en composantes principales (ACP). Les destinations seraient alors classées en fonction des scores établis.

\section{BIBLIOGRAPHIE}

Valérie ANGERON et Pascal SAFFACHE, "Les petites économies insulaires et le développement durable : des réalités locales résilientes ?”, Études caribéennes, n 11, décembre 2008 [en ligne]. Cédric AUDEBERT, L’Insertion socio-spatiale des Haïtiens à Miami, 2006, L'Harmattan.

Rémi BARRÉ, Valeria HERnÁNDEZ, Jean-Baptiste MEYER et Dominique VINCK, Diasporas scientifiques. Comment les pays en développement peuvent-ils tirer parti de leurs chercheurs et de leurs ingénieurs?, IRD, 2003.

Junia BARREAU, "Investissements directs étrangers : la difficile équation haïtienne", Canal + Haïti, 2012 www.canalplushaiti.net/?p=2862 
Brigitte BERTONCELLO, "Diaspora, développement et urbanisation : des Burkinabè de l'étranger acteurs des projets urbains de Zaca et Ouaga 2000 à Ouagadougou (Burkina Faso)", Annales de géographie, vol. 5, nº 675, 2010.

Jim BlyTHE, Key Concepts in Marketing, Sage, 2009.

F. BONNET, “Haïti : l'île de toutes les tragédies", Marianne, n 65, 2010.

Chantal BORDES-BENAYOUN, "Les diasporas, dispersion spatiale, expérience sociale”, Autrepart, $\mathrm{n}$ - 22, 2002.

Joseph BRAM, Lenguaje y sociedad, Paidos, 1967.

Michel BRUNEAU, "Les territoires de l'identité et la mémoire collective en diaspora", L'Espace géographique, vol. 35, n 4, 2006.

Romain CRUSE, Géopolitique et migrations en Haïti. Essai sur les causes de l'émigration haïtienne et sur l'utilisation des migrants, Publibook, 2012.

Marvel DANDIN, “Pour une intégration efficace de la diaspora au développement d'Haïti”, Rencontre, $\mathrm{n}^{\circ} 24-25,2012$.

Joseph Ronald DAUTRUCHE, "Tourisme culturel et patrimoine remodelé. Dynamique de mise en valeur du patrimoine culturel immatériel en Haïti”, Ethnologies, vol. 35, nº 1, 2013.

Joseph Ronald DAUTRUCHE, Culture, patrimoine et tourisme en Haïti. Construction et dynamique de reconstruction d'une destination touristique, thèse de doctorat, Université Laval, 2013.

Arjan DE HAAN, "Migrants, livelihood and rights: The relevance of migration in development policies", Social Development Working Paper, $\mathrm{n}^{\circ} 4$, Londres, Department for International Development, 2000.

Fred DERVIN, "Multi-positioning of the self and the other in a radio documentary about the work of an anthropologist: (Co-)constructing truth and silence in a case of incest", dans Constructing Identity in Interpersonal Communication/Construction identitaire dans la communication interpersonnelle/Identitätskonstruktion in der interpersonalen Kommunikation, Helsinki, Société néophilologique, 2010.

Philippe DEWITTE, “Des citoyens à part entière, ou entièrement à part ?", Diasporas caribéennes, $\mathrm{n}$ - 1237, 2002.

Guichard DORÉ, Politique de formation professionnelle et d'emploi en Haïti. Le cas du secteur du tourisme (1980-2010), thèse de doctorat, Université Paris-Est, 2010.

Éric DUBESSET, “Penser autrement l'identité régionale caribéenne”, Études caribéennes, $\mathrm{n}^{\circ} 21$, avril 2012 [ DOI : 10.4000/etudescaribeennes.5739].

Stéphane DufoIx, Les Diasporas, coll. “Que sais-je ?”, Puf, 2003.[5:

Louis DuPONT, "Co-intégration et causalité entre développement touristique, croissance économique et réduction de la pauvreté : cas de Haïti”, Études caribéennes, décembre 2009 [ http://etudescaribeennes.revues.org/3780 ]

Berthony DuPONT, “Ne soyons plus un peuple objet”, Courrier international, n 1003, 2010.

Brice Duthion et Jean-Luc Michaud, "Préface”, dans Chris Cooper et C. Michael Hall, Le Tourisme aujourd'hui : une approche internationale, De Boeck, 2011.

Gilbert ELBAZ, "L'hybridation transnationale des diasporas", Études caribéennes, n 16, 2010a [ http://etudescaribeennes.revues.org/4627 ].[.틀 
Gilbert ElBAZ, “Diasporas protéiformes”, Études caribéennes, n 16, 2010b [ http:// etudescaribeennes.revues.org/4758 ].

Alain FAURE, “Gouvernements intercommunaux et ressources politiques. L'identité territoriale pour énoncer des priorités d'action publique ?”, dans Denis-Constant Martin (dir.), L'Identité en jeux. Pouvoirs, identifications, mobilisation, Karthala, 2010.

Marjolein C. GROOT et Patt GibBons, “Diasporas as 'agents of development' : Transforming brain drain into brain gain ? The Dutch example", Development in Practice, vol. 17, n 3, 2007.

Michael Hammond et Jerry Wellington, Research Method: The Key Concepts, Routledge, 2013.

Kam HUNG, Honggen XIAO et Xiaotao YANG, "Why immigrants travel to their home places: Social capital and acculturation perspective", Tourism Management, $\mathrm{n}^{\circ}$ 36, 2013.

Evans JADotTE, Brain Drain, Brain Circulation and Diaspora Networks in Haiti, UNCTAD, Background Paper, 2012.

Lesly Jules et Kareen THERMIL-LAPLANCHE, "Le Tourisme en Haïti : diagnostic, stratégies, perspectives", actes du colloque Tourisme et développement durable (Université Quisqueya, Haïti), Ceregmia, 2006.

Tracy KIDDER, “Miser sur le peuple haïtien”, Courrier international, n 1003, 2010.

Maximiliano KoRSTANJe, "The influence of language in the travel agencies name: The case of Buenos Aires", The Journal of Hospitality and Tourism, vol. 10, nº 1, 2012.

Maud LAËTHIER, Être haïtien et migrant en Guyane française, thèse de doctorat en anthropologie sociale et ethnologie, EHESS Paris, 2007.

Gilles LARIVIERE et Jocelyn JusSAume, "Émergence des nouvelles formules en hôtellerie", Teoros, vol. 23, n 3, 2004 [ http://teoros.revues.org/720 ].

J. B. LEWIS, "The development of rural tourism", Parks and recreation, vol. 33, n 9, 1998.

Elisabetta LODIGIANI, “Diaspora externalities and technology diffusion”, Économie internationale, vol. $3, \mathrm{n}^{\circ} 115,2008$.

Denis-Constant MARTIN (dir.), L'Identité en jeux. Pouvoirs, identifications, mobilisation, Karthala, 2010.

Jean-Baptiste MEYER et Mercy BRown, "Scientific diasporas : A new Approach to the brain drain", Discussion Paper, UNESCO-MOST, n 41, 1999 [en ligne : http://www.unesco.org/most/meyer.htm].

Stéphanie MELYON-REINETTE, “De la dédiasporisation des jeunes Haïtiens à New-York”, Études caribéennes, 2010 [ http://etudescaribeennes.revues.org/4628 ].

Stéphanie MELYON-REINETTE, Haïtiens à New York City : Entre Amérique noire et Amérique multiculturelle , L'Harmattan, 2009.

Kathleen NEWLAND et Carylanna TAYLOR, Heritage Tourism and Nostalgia Trade: A Diaspora Niche in the Development Landscape, Migration Policy Institute, 2010.

Manuel OROZco, Understanding the remittance economy in Haïti, World Bank, 2006.

Manuel OROZCO, Remittances to Latin America and the Caribbean: Issues and perspectives on development , report commissioned by the Organization of American States, 2004. 
Bénédique PAUL, "Migration et pauvreté en Haïti : impacts économiques et sociaux des envois de fonds sur l'inégalité et la pauvreté ?", communication à la journée thématique Envois de fonds, inégalité et pauvreté dans les pays en développement (16 octobre 2008, université Bordeaux 4), 2008.

Bénédique PAUL, L'Haïtianité. Institutions et identité en Haïti, TheBookEdition.com, 2009.

Bénédique PAUL, “Diaspora et Développement en Haïti. Vers une participation intelligente et efficiente", communication au 2012 Global Haitian Diaspora Unity Congress : Unity for Results : in and out of Haiti (Haitian Diaspora Federation, Washington DC, du 18 au 20 octobre), 2012b.

Bénédique PAUL et Thébeau Michel, “Comment juguler les limitations financières des universités haïtiennes ?", Haïti Perspectives, Grahn, vol. 2, nº 1, 2013a.

Bénédique PAUL et Thébeau Michel, "Addressing the problem of human and financial resources mobilization in Haitian universities: Propositions for better proactive institutional and managerial strategies", Research Journal of Business Management and Accounting, vol. 2, n 3, $2013 \mathrm{~b}$.

Angel PANIAGUA, "Urban-rural migration, tourism entrepreneurs and rural restructuring in Spain", Tourism Geographies, vol. 4, n 4, 2002.

Adela Pellegrino, "Trends in international migration in Latin America and the Caribbean", International Social Science Journal, vol. 165, 2000.

Naomi Pocock et Alison Mcintosh, “Long-term travellers return, 'home'?”, Annals of Tourism Research vol. 42, 2013.

Olivier Pulvar, "Mémoire, médiatisation et construction des identités", Études caribéennes, $\mathrm{n}^{\circ}$ 5, 2006 [en ligne].

Omar SAGHI, "Exil, errance, vacances : les tourismes diasporiques", EspacesTemps.net, 2011 [ http://www.espacestemps.net/articles/exil-errance-vacances-les-tourismes-diasporiques/ ].

Regina SCHEYVENS, "Poor cousins no more: Valuing the development potential of domestic and diaspora tourism", Progress in Development Studies, vol. 7, $\mathrm{n}^{\circ} 4,2007$.

Hugues SÉRAPHIN, Le Tourisme : l'ouverture pour le peuple de Toussaint ? Précis sur le tourisme en Haïti, Publibook, 2014a.

Hugues SÉRAPHIN, “Les jeux d'influences dans le tourisme : cas d'Haïti”, Journal of Haitian Studies, vol. $20, \mathrm{n}^{\circ} 2,2014 \mathrm{~b}$.

Hugues SÉRAPHIN, “Hispaniola: The future tourism destination of the Caribbean?", Journal of Tourism Consumption and Practice, vol. 3, $\mathrm{n}^{\circ}$ 2, 2011.

Hugues SÉRAPHIN, “Quel avenir pour le tourisme en Haïti ?”, Espaces tourisme \& loisirs, n² 281, 2010.

Lawrence SILVER, Robert STEVEns, Bruce WrENn et David Loudon, The Essentials of Marketing Research, Routledge, 2013 (3 édition).

Jean-Marie THÉODAT, “L'endroit et l'envers du décor : la 'touristicité' comparée d'Haïti et de la République dominicaine" Revue Tiers Monde, t. 45, n 178, 2004.

A. R. THERNIL, "Perceptions of Haitians toward tourism development in rural Haïti", Symposium sur la recherche dans le domaine des loisirs (Ball State University, USA), 2004.

Ian THOMson, Bonjour Blanc: A Journey through Haïti, Vintage, 2004 [1991].

Jérôme VANDENBUSSCHE, Philippe AGHion et Costas MEGHIR, "Growth, distance to frontier and composition of human capital", Journal of Economic Growth, vol. 11, n 2, 2006. 
Roselyne DE VILLANOVA, “Espace de l'entre-deux ou comment la mobilité des immigrés recrée du territoire", L'Homme et la société, vol. 3, n 165-166, 2007.

Tatiana WAH, “Engaging the Haitian Diaspora”, The Cairo Review of Global Affairs, avril 2013.

\section{NOTES}

1. Bulletin trimestriel de statistiques touristiques, vol.2, MTIC, Service des statistiques et de recherches, 2011.

2. Voir aussi le site de Canal+ Haïti [ www.canalplushaiti.net ].

3. Radio Canal+ Haïti, 2014.

4. Julien Frandley, Le Nouvelliste, 12 novembre 2014 [ http://lenouvelliste.com/lenouvelliste/ article/138057/Investir-et-sinvestir-en-Haiti-Un-acte-de-foi-Jerry-Tardieu-fait-salle-comble-aMoca-Cafe ].

5. "L'influence des langues sur le nom des agences de voyages : étude de cas de Buenos Aires."

6. The Haitians Diaspora in the United States, Migration Policy Institute, 2014.

\section{RÉSUMÉS}

L'identité haïtienne, ou encore l'haïtianité, s'exprime fortement au sein de la diaspora. Dans cet article, nous avons cherché à comprendre dans quelle mesure cette expression identitaire se traduit en moteur de développement territorial en Haïti, avec un accent particulier porté sur le secteur du tourisme. Nous parvenons à la conclusion que les conséquences positives de l'action diasporique dans la dynamique territoriale en Haïti apparaissent comme des atouts qui méritent d'être pris en compte dans une démarche de planification du développement touristique. L'un des dangers qui guettent cependant le développement de cette activité en Haïti est le développement d'enclaves touristiques.

The Haitian identity, also called "haitianity" is notably expressed in the diaspora. In this paper, our objective is to figure out how this haitianity can be used as a boost for the territorial development of Haiti (with a particular focus on the tourism industry). The positive impacts of the diaspora on the Haitian territorial dynamic deserve to be taken into consideration particularly when it comes to the development of the tourism sector. However, one of the main issues with the development of this activity in Haiti is the creation of enclaves.

\section{INDEX}

Mots-clés : haïtianité, diaspora, développement territorial, tourisme, enclave

Keywords : haitianity, diaspora, territorial development, tourism, enclave 


\section{AUTEURS}

\section{HUGUES SÉRAPHIN}

Maître de conférences, université de Winchester (Angleterre)

hugues.seraphin@winchester.ac.uk

\section{BÉNÉDIQUE PAUL}

Enseignant-chercheur et directeur adjoint du Centre de recherche en gestion et en économie du développement (CREGED) ; enseignant-chercheur associé au Centre de recherche et d'appui aux politiques urbaines (CRAPU), Université Quisqueya (uniO), Haïti benediquep.paul@uniq.edu.ht 